The mean disease duration was $6,4 \pm 5,8$ years $(0,5-30)$. The observation period was 12 years. All pts received prednisolone at a dose of $11,7 \pm 4,8 \mathrm{mg} /$ day, and 73 patients $(49 \%)$ received immunosuppressants at inclusion. The indications for the appointment of RTX were ineffectiveness or impossibility of standard therapy and a severe course of the disease with high activity and unfavorable prognosis factors. $\mathrm{AE}$ were assessed and recorded by a physician at a hospital immediately after the infusion of RTX, then by patient reported outcome during the observation period. Severe $A E$ were defined as those that required hospitalization for more than 24 hours, exacerbation of the disease requiring therapy, malignancies, life-threatening situations. All causes of death were considered, regardless of treatment.

Results: The mean follow-up period after the first infusion of RTX was 5,6 $\pm 2,6$ years [834,4 patient-years (PY)]. Pts received a mean of 3,4 courses of RTX (1-10). The cumulative mean dose of RTX was $3,2 \pm 2,4 \mathrm{gr}(0,5-11)$. AE were reported in 77 patients $(52 \%)$, the overall frequency of $A E$ was $9,3 / 100 \mathrm{PY}(95 \%$ Confidence Interval (CI) 8-11). The highest frequency of all AE was observed in the first 2-6 months after the first infusion of RTX, however these were mainly mild AE $(71 \%)$. There was a decrease of $\mathrm{AE}$ in the follow-up period (3,4/100 PY, 95\% Cl 2,4-4,9 - at the period from 3 to 10 course of RTX). The overall incidence of serious AE was 2,22/100 PY $(95 \% \mathrm{Cl} 1,4-3,5)$. The specter of serious $\mathrm{AE}$ included: pneumonia in $7 \mathrm{pts}$, infusion reactions in 5, as well as in one case: cerebral ischemia, acute pancreatitis, allergic pneumonitis, lymphoma of pharynx, purulent arthritis, lower limb vein thrombosis, pulmonary embolism of small arteries. The most frequent $A E$ were infections $(n=53)$, with no serious opportunistic infections reported. The overall incidence of all infections was 6,4/100 PY (95\% Cl 4,9-8,3), serious infections - 1,32/100 PY $(95 \% \mathrm{Cl} 0,7-2,4)$. The level of immunoglobulin $\mathrm{G}$ during follow-up period decreased from $12,9 \pm 4,9$ to $10,1 \pm 3,4 \mathrm{~g} / \mathrm{l}(\mathrm{p}=0,0001)$, but remained within normal limits. Infusion reactions occurred in 15 pts $(1,8 / 100 \mathrm{PY}, 95 \% \mathrm{Cl} 1-3)$. Other $\mathrm{AE}$ were observed in 9 pts $(6 \%)(1,1 / 100$ PY, $95 \%$ Cl 0,53-2,12). Sixteen deaths were recorded $-10,7 \%$ or $1,91 / 100 \mathrm{PY}, 95 \% \mathrm{Cl} 1,2-3,1$. In most cases, pts died from the progression of the major organ failure. The causes of death were: progression of the interstitial lung disease (ILD) in 4 pts, heart failure associated with SSc cardiomyopathy (2), renal crisis (4), pulmonary arterial hypertension and ILD (2), pneumonia (2), sepsis after tooth extraction (1), acute pulmonary embolism (1).

Conclusion: In our study, we considered the overall safety profile of RTX in SSc as favorable. It was similar to the $\mathrm{AE}$ profile in other autoimmune diseases treated with RTX. With an increase of the cumulative dose of RTX, there was no increase in AE. RTX could be considered as a relatively safe drug for the complex therapy of SSc when standard therapy is ineffective or impossible.

Disclosure of Interests: None declared

DOI: 10.1136/annrheumdis-2021-eular.1325

\section{AB0424 NUTRIENT DEFICIENCIES IN SYSTEMIC SCLEROSIS: A SYSTEMATIC REVIEW}

A. Nguyen ${ }^{1}$, Z. Mcmahan ${ }^{2}$, E. Volkmann ${ }^{3} .{ }^{1}$ David Geffen School of Medicine at UCLA, Medicine, Los Angeles, United States of America; ${ }^{2}$ Johns Hopkins University School of Medicine, Medicine - Rheumatology, Baltimore, United States of America; ${ }^{3}$ David Geffen School of Medicine at UCLA, Medicine Rheumatology, Los Angeles, United States of America

Background: Malnutrition is a critical concern in patients with systemic sclerosis (SSc). ${ }^{1,2}$ However, the extent and types of nutrient deficiencies in SSc remain unclear.

Objectives: 1) To identify the nutrient deficiencies commonly reported in SSc; 2) To evaluate associations between specific nutrient deficiencies and SSc subtype and clinical manifestations.

Methods: We conducted a systematic review of all published reports on SSc and nutrition in PubMed from its inception to August 2020. Clinical trials, observational studies, and case series (with $\geq 20$ cases) containing data on nutritional deficiency and SSc were included. We followed the Preferred Reporting Items for Systematic Reviews and Meta-Analyses (PRISMA) for reporting our findings. Two reviewers (ADN and ERV) studied the titles and abstracts of all search results with pre-specified inclusion and exclusion criteria (Figure 1).

Results: Among 790 retrieved publications, 35 full-length articles and 3 abstracts met the inclusion criteria. Included studies took place across multiple geographic locations and included patients with both diffuse and limited cutaneous SSc. Vitamin $D$ deficiency was the most commonly reported deficiency described in SSc, followed by vitamin B12, folate, selenium, zinc, and iron (Table 1). In addition, some small studies found deficiencies in thiamine, pyridoxine, alpha-tocopherol, and carotene. Interstitial lung disease was associated with vitamin $\mathrm{D}$ deficiency and elevated homocysteine (Hcy), while pulmonary hypertension was associated with elevated Hcy. Vitamin D deficiency was also associated with increased modified Rodnan skin score (mRSS) and increased risk of SSc-related organ involvement. Conclusion: Nutrient deficiencies are common in SSc and are associated with specific SSc features. Routine screening for nutrient deficiencies may lead to early detection of malnutrition. Future studies are needed to understand how nutritional interventions affect patient outcomes in SSc.

REFERENCES:
[1] Harrison E, Herrick AL, McLaughlin JT, Lal S. Malnutrition in systemic sclerosis. Rheumatology. 2012;51(10):1747-1756. doi:10.1093/rheumatology/kes160

[2] Baron M, Hudson M, Steele R, et al. Malnutrition is common in systemic sclerosis: Results from the Canadian Scleroderma Research Group database. J Rheumatol. 2009;36(12):2737-2743. doi:10.3899/jrheum.090694

Figure 1. Article selection for systematic review.

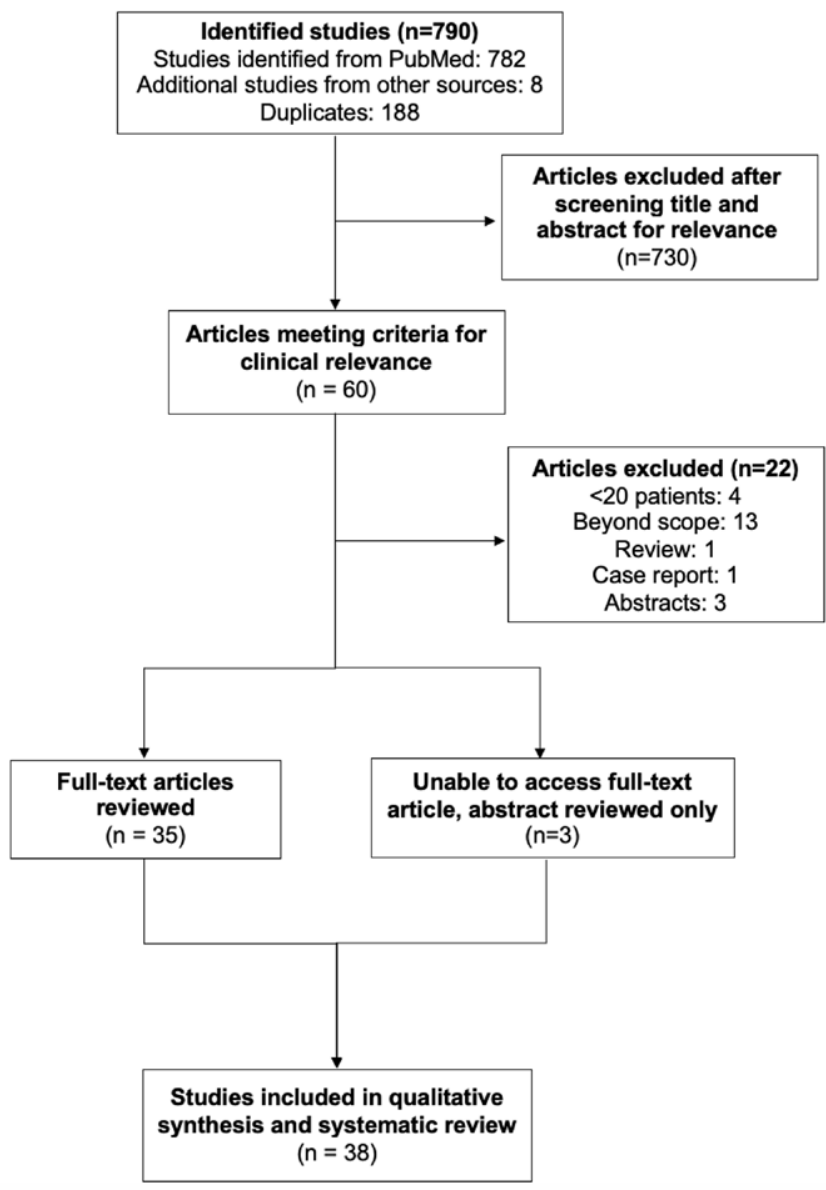

Table 1. Nutrient deficiencies and their clinical correlates in SSc.

\begin{tabular}{|c|c|c|c|}
\hline & $\begin{array}{c}\text { Number of } \\
\text { studies that found } \\
\text { deficiency }^{\mathrm{a}}\end{array}$ & $\begin{array}{c}\text { Number of studies } \\
\text { that did not find } \\
\text { deficiency }\end{array}$ & $\begin{array}{l}\text { Clinical correlates } \\
\text { of nutrient } \\
\text { deficiency }\end{array}$ \\
\hline Vitamin D & 23 & 0 & $\begin{array}{c}\text {-Increased } \\
\text { risk of SSc- } \\
\text { related organ } \\
\text { involvement } \\
\text {-ILD } \\
\text {-Higher mRSS }\end{array}$ \\
\hline Homocysteine $^{b}$ & 5 & 4 & $\begin{array}{l}\text {-ILD } \\
\cdot \mathrm{PH}\end{array}$ \\
\hline Vitamin B12 & 3 & 4 & -None identified \\
\hline Folate & 3 & 2 & -None identified \\
\hline Vitamin C & 2 & 0 & -.None identified \\
\hline Selenium & 5 & 0 & $\begin{array}{l}\text {-Increased } \\
\text { risk of SSc- } \\
\text { related organ } \\
\text { involvement }\end{array}$ \\
\hline Zinc & 3 & 1 & -None identified \\
\hline Iron & 1 & 1 & -None identified \\
\hline Copper & 0 & 2 & -None identified \\
\hline Other nutrients & & & $\begin{array}{l}\text {-None identified } \\
\text { for all nutrients }\end{array}$ \\
\hline Thiamine & 1 & 0 & \\
\hline Pyridoxine & 1 & 0 & \\
\hline Alpha-tocopherol & 1 & 0 & \\
\hline Carotene & 1 & 0 & \\
\hline
\end{tabular}

${ }^{\mathrm{a}}$ Deficiency defined as below normal levels based on study reference ranges ${ }^{\mathrm{b}}$ Studies looked for elevation versus no elevation of homocysteine in $\mathrm{SSc}^{\mathrm{c}}$ Some studies found significant correlation while others did not 
Acknowledgements: I have no acknowledgements to declare.

Disclosure of Interests: None declared

DOI: 10.1136/annrheumdis-2021-eular.1420

\section{AB0425 1 CLINICAL AND PATHOLOGICAL FEATURES OF BREAST CANCER IN PATIENTS WITH SYSTEMIC SCLEROSIS: PRELIMINARY DATA FROM THE SCLERO-BREAST STUDY}

A. Spinella ${ }^{1}$, A. Toss $^{2}$, C. Isca ${ }^{2}$, C. Vacchi ${ }^{1}$, A. Iannone ${ }^{3}$, L. Magnani ${ }^{4}$, P. Castrignanò $^{1}$, M. De Pinto ${ }^{1}$, C. Laura ${ }^{2}$, A. Maiorana $^{5}$, C. Salvarani ${ }^{1,4}$, M. Dominici ${ }^{2}$, D. Giuggioli ${ }^{1}$. ${ }^{1}$ University of Modena and Reggio Emilia, Scleroderma Unit, Rheumatology Unit, Modena, Italy; ${ }^{2}$ University of Modena and Reggio Emilia, Department of Oncology and Hematology, Modena, Italy; ${ }^{3}$ University of Modena and Reggio Emilia, Department of Surgery, Medicine, Dentistry and Morphological Sciences with Transplant Surgery, Oncology and Regenerative Medicine Relevance, Modena, Italy; ${ }^{4}$ AUSL-IRCCS of Reggio Emilia, Italy, Rheumatolgy, Reggio Emilia, Italy; ${ }^{5}$ University of Modena and Reggio Emilia, Pathology Unit, Modena, Italy

Background: Systemic Sclerosis (SSc) is a rare and life-threatening connective tissue disease characterized by vascular dysfunction, specific autoimmune abnormalities and fibrosis of the skin and internal organs. Previous studies have shown a 1.5-fold increase in cancer risk in SSc patients compared with the general population, including breast cancer $(\mathrm{BC})$. The relationship between $\mathrm{BC}$ and SSc has long been discussed but past research has been contradictory and inconclusive on this topic.

Objectives: The aim of our project was to analyze clinical and pathological characteristics of BC developed by SSc subjects and possible correlations with scleroderma features. Here we present the preliminary data from the Sclero-Breast study.

Methods: Our observational retrospective multicenter study enrolled 33 SSc women with a personal history of BC identified at two Rheumatology/SSc Units in the north of Italy between January 2017 and December 2019 (Ic/dcSSc 23/9, 1 unknown; mean age at SSc onset 57 years, range 32-73). All patients underwent general and instrumental assessment: smoking habits; presence of skin ulcers, calcinosis, teleangectasia; presence of gastro-intestinal and kidney involvement; interstitial lung disease (at HR-CT); pulmonary function tests; ECG abnormalities; echocardiographic assessment of pulmonary arterial hypertension (PAH); videocapillaroscopic pattern; autoantibody profile; exposure to immunosuppressive and vasoactive therapies; status at last follow-up evaluation and cause of death. Clinical and pathological characteristics of BC were also evaluated: age at diagnosis; menopausal status; histotype; hormone receptor status; MIB1, HER2 expression; clinical and pathological stage at diagnosis; metastatic sites; type of loco-regional treatment (surgery and radiotherapy); type of systemic treatment (neoadjuvant/adjuvant chemotherapy and endocrine treatment); other cancers and time from diagnosis of the first disorder to the second one.

Results: A total of $54.5 \%$ of subjects developed BC before SSc (median interval of 5 years), whereas $45.5 \%$ of patients developed BC after SSc (median delay of 8 years). $54.5 \%$ of patients showed interstitial lung disease and the cause of death of the 6 deceased subjects was $\mathrm{PAH}$. A significant association $(p<0.05)$ was observed between the use of immunosuppressive therapy and diffuse skin extension, negative ACA, positive Anti-Scl-70 and interstitial lung disease, but not with BC status. $93.1 \%$ of patients were diagnosed with an early-stage tumor, $70.8 \%$ of invasive carcinomas with a low MIB-1, $8.3 \%$ with a tubular histotype, while $42.8 \%$ presented with a Luminal A-like tumor. $66.6 \%$ underwent breast conserving surgery and $55.5 \%$ RT after surgery. $40 \%$ of patients developed interstitial lung disease after RT and $20 \%$ dcSSc.

Conclusion: According to our preliminary data, SSc patients developed BC at good prognosis, suggesting a de-escalation strategy of cancer therapies. On these grounds, a proper screening is mandatory in order to allow for early cancer detection in SSc patients. Further investigations on larger numbers of patients are needed. First of all, they would further clarify the intriguing relationship between BC and SSc. Secondly, they would help to explore the common biological and molecular pathways at the basis of these two disorders, with the aim to improve $\mathrm{BC}$ diagnosis and prognosis and to personalize oncological targeted treatments in this subset of fragile patients.

Disclosure of Interests: None declared

DOI: 10.1136/annrheumdis-2021-eular.1450
AB0426

CANCER SCREENING IN IDIOPATHIC INFLAMMATORY MYOPATHIES: TEN YEARS EXPERIENCE FROM A SINGLE CENTER

E. Trallero-Araguás ${ }^{1}$, A. Gil-Vila ${ }^{2}$, X. Martínez-Gómez ${ }^{3}$, M. Alvarado-Cardenas ${ }^{2}$, M. Simo-Perdigó ${ }^{4}$, J. Ros ${ }^{5}$, I. Pinal-Fernandez ${ }^{6}$, A. Selva-O'callaghan7. ${ }^{1}$ Vall d'Hebron Hospital, Rheumatology, BARCELONA, Spain; ${ }^{2}$ Vall d'Hebron Hospital, Medicine, Barcelona, Spain; ${ }^{3}$ Vall d'Hebron Hospital, Preventive Medicine and Epidemiology, Barcelona, Spain; ${ }^{4}$ Vall d'Hebron Hospital, Nuclear Medicine, Barcelona, Spain; ${ }^{5}$ Vall d'Hebron Hospital, Medical Oncology, Barcelona, Spain; ${ }^{6}$ National Institutes of Health, Rheumatology, Bethesda, Spain; ${ }^{2}$ Vall d'Hebron Hospital, Medicine, Barcelona, Spain

Background: There is a well-recognized association between cancer and myositis, so cancer screening at diagnosis is recommended.

Objectives: We aim to report the results of our cancer screening strategy and to ascertain the reliability of using PET/CT to identify cancer-associated myositis (CAM) in a large cohort of patients with myositis from a single center over 10 years.

Methods: This retrospective observational study included all patients diagnosed with any type of myositis except for inclusion body myositis. Cancer screening strategy was individualized according to clinical and serological data, including $\mathrm{PET} / \mathrm{CT}$ as the main test to detect occult cancer (OC). Procedures derived from a positive PET/CT were registered. Qualitative data expressed as percentages, and quantitative data as the median with the interquartile range were analyzed. A ROC curve was used to estimate the reliability of PET/CT for CAM diagnosis. Results: Seventy-seven out of 131 patients underwent a PET/CT for OC screening. The performance of the PET/CT in patients with myositis at disease onset yielded an area under the curve ROC of 0.87 (0.73-0.97) for CAM diagnosis. Invasive procedures in 7 (9\%) patients without a final diagnosis of cancer did not cause derived complications. Patients not evaluated for OC did not develop cancer after a median follow-up of 3.3 years (1.7-6.7).

Conclusion: Cancer screening strategy should be individualized. PET/CT at myositis onset seems to be an efficient approach to rule out CAM. This practice does not seem to significantly increase harm to patients related to the additional tests needed to clarify inconclusive results.

Disclosure of Interests: None declared

DOI: 10.1136/annrheumdis-2021-eular.1457

\section{AB0427 \\ ANTI-NOR90 AUTOANTIBODIES: FAVORABLE OR UNFAVORABLE PROGNOSIS?}

$\underline{\text { C. Valero }}^{1}$, J. P. Baldivieso ${ }^{1}$, I. Llorente ${ }^{1}$, E. F. Vicente-Rabaneda ${ }^{1}$, L. Esparcia Pinedo. $^{2}$, R. Garcia de Vicuna ${ }^{1}$, M. A. Alfranca ${ }^{2}$, S. Castañeda ${ }^{1} .{ }^{1}$ Hospital Universitario de la Princesa, Rheumatology, Madrid, Spain; ${ }^{2}$ Hospital Universitario de la Princesa, Immunology, Madrid, Spain

Background: Anti-NOR 90 autoantibodies (anti-NOR90 Ab) are autoantibodies that target nucleolar transcription factor 1 or hUBF, involved in transcription of RNA polymerase I. These autoantibodies have been detected in $6.1 \%$ of patients with Systemic Sclerosis (SSc), but their clinical or prognostic significance has not been clearly defined. Anti-NOR90 Ab have been mostly associated with limited scleroderma with mild organ involvement and can also be found in other rheumatic diseases such as rheumatoid arthritis, systemic lupus erythematosus or Sjogren's syndrome.

Objectives: The aim of this study was to identify the main clinical characteristics of patients with positive anti-NOR90 in our Centre.

Methods: This is a retrospective, descriptive, cross-sectional study of all patients with positive anti-NOR90 Ab between January 2013 and December 2020 in a single center. Autoantibodies testing was performed using Euroimmun EUROLINE SSc profile IgG autoAb assay kit. Patient demographics, clinical characteristics, associated diagnoses, laboratory and immunological findings were collected. Results: We identified a total of 26 patients with at least a positive value for antiNOR90 Ab (Table 1). In most cases anti-NOR90 patients were ANA positive, predominantly with nucleolar pattern and coexisted with other SSc autoantibodies. 12 patients had rheumatic diseases and two had SSc, both with limited cutaneous SSc and absence of organ involvement. 14 patients had no definite diagnosis. Clinical features of anti-NOR90 patients are represented in Figure 1. Five patients presented Raynaud's phenomenon, two cases with pathological nailfold capillaroscopy and one patient had SSc. There was no patient with skin ulcers, calcinosis, interstitial lung disease or pulmonary hypertension. Four patients had gastroesophageal reflux disease and one patient presented antral vascular ectasia. Six patients developed some neoplasm. 